\title{
Review Article \\ Determination of Glucose Levels during Dialysis Treatment: Different Sensors and Technologies
}

\author{
Stefano Sbrignadello, Giovanni Pacini, and Andrea Tura \\ Metabolic Unit, CNR Institute of Neuroscience, Corso Stati Uniti 4, 35127 Padua, Italy \\ Correspondence should be addressed to Andrea Tura; tura@isib.cnr.it
}

Received 11 August 2015; Revised 30 October 2015; Accepted 2 November 2015

Academic Editor: Michael J. Schöning

Copyright ( 2016 Stefano Sbrignadello et al. This is an open access article distributed under the Creative Commons Attribution License, which permits unrestricted use, distribution, and reproduction in any medium, provided the original work is properly cited.

The measurement of glycemia in subjects with renal failure, thus treated with hemodialysis, or peritoneal dialysis, is clinically relevant, since glucose levels may influence the determination of other solutes, such as creatinine, as well as some ions, such as sodium, whose degree of removal during dialysis sessions should be controlled carefully. Also, glucose levels should be controlled to avoid possible events of hypoglycemia during the treatment, especially in diabetic subjects. Indeed, even cases of hypoglycemic coma are documented. The glucose measurement during the dialysis treatment can be performed with different sensors and technologies: for instance, with traditional glucose meters, with instruments for continuous glucose monitoring, or with optical sensors. The aim of this review study was to analyze these different approaches and briefly discuss possible advantages and limitations.

\section{Introduction}

The number of subjects worldwide with chronic kidney disease has been found to increase year after year, and the main driving factors behind this alarming data seem to be the increasingly aged global population and the epidemic of type 2 diabetes mellitus [1]. End-stage renal disease and its standard of care, that is, renal replacement therapy (including dialysis and/or kidney transplantation), result in a huge economic and collective fee. Incidence rates for renal replacement therapy all over the world range from a minimum of 12 to a maximum of 455 subjects per million of population, with a median of 130 , and with an increase of $6 \%$ to $7 \%$ annually. Although affecting up to $0.03 \%$ of the total population in industrialized countries, end-stage renal disease consumes up to $3 \%$ of annual healthcare resources [2]. Thus, the financial and health burden of renal replacement therapy on society demands technology capable of maximizing efficiency in treatment in terms of both costs and clinical outcomes.

The importance of glycemic control is widely established, and it is a relevant component in managing individuals with critical illness and with complications of diabetes mellitus [3]. Inappropriate glycemic control is a risk factor for micro and macrovascular diseases in diabetic people (both types 1 and 2). Also, the quality of glycemic control is known to be a relevant factor for the level of progression of diabetic nephropathy. On the other hand, the glycemic control in predialysis condition is determinant for progression of other diabetic complications. As an example, constant high glucose exposure drives tissue and plasma proteins to increase levels of advanced glycation end products, which can lead to accelerated atherosclerotic cardiovascular disease [4].

During dialysis treatment, hyperglycemia can also lead to hyponatremia: this happens when the serum contains an excessive amount of additional osmoles (such as glucose), which increases the effective osmolality and reduces the serum sodium concentration by attracting water from the intracellular compartment. The serum becomes hypotonic, with the final result that both sodium concentration and effective osmolality become low. Hyponatremia can then lead to a wide spectrum of clinical symptoms, from slight to severe or even life threatening, and it is associated with increased mortality, morbidity, and duration of hospitalization [5]. On the other hand, high glycemic variability, compared to constant hyperglycemia, seems to have even more dangerous cardiovascular effects, due to its high positive correlation with 
the urinary excretion rate of isoprostane 8-iso-prostaglandinF2 $\alpha$, which is a well-known oxidative stress marker [6].

The opposite risk, in subjects under dialysis treatment, is hypoglycemia [7]. Indeed, hypoglycemia is not uncommon during dialysis, and it can even lead to coma and death. In fact, hypoglycemic events increase with intensive treatment, and in the presence of cardiovascular diseases it can cause fatal dysrhythmia $[8,9]$.

The glucose measurement may be also important for evaluating, indirectly, the effectiveness of peritoneal dialysis treatment, since glucose levels have been reported to interfere with the measurement of creatinine concentration in some creatinine assays. Measurement of creatinine in peritoneal dialysis fluid is required to assess the permeability of the peritoneum or for calculation of peritoneal creatinine clearance, which is a marker of the adequacy of dialysis [10].

In summary, determination of glucose levels during dialysis treatment is clinically important [11]. The aim of this review is to present and analyze the different sensors, technologies, and approaches for the glucose measurement during dialysis. For searching the scientific literature, we used Scopus search engine, using the following keywords properly combined: glucose, glycemia (or glycaemia), spectrometer (or spectrophotometer, or spectroscopy), infrared, NIR or IR, optical, electromagnetic, ultraviolet or UV, fluorescence, measure (or measurement), monitor (or monitoring), meter, assess (or assessment), evaluate (or evaluation), assay, hemodialysis (or haemodialysis), and peritoneal dialysis. From the initial research, we identified about 200 articles, subsequently reduced to 47 after inspection of the abstract. After reviewing the full text, we chose 26 articles to be included in this review study. Main exclusion criterion was the limited information available on sensors and technologies used. To our knowledge, this is the first review study focused on sensors and technologies for glucose measurement during dialysis treatment.

\section{Glucose Determination with Traditional Approaches}

One possibility for glucose determination during dialysis is using a traditional glucose meter or laboratory glucose analyzer (Figure 1). Depending on the experiments conducted in the examined studies, the methods used were the traditional strip method (electrode-glucose oxidase biosensor) or the standard procedures of medical laboratory analysis, which represent the gold standard measure of blood glucose.

The first in-depth evaluation and application of blood glucose measurements in subjects during dialysis session were performed, to our knowledge, by Wason et al. [12] with the classic sensor enzyme strip method (Chemstrip bG, BioDynamics Boehringer-Mannheim, Indiana, USA). Tests were performed before and after dialysis. However, glucose sensor strips are not extremely accurate: errors in strip methods may reach $15 \%$ [13].

In the study [14], Bewley et al. used again a traditional method to control hemodialysis-induced changes in plasma glucose concentrations (in diabetic subjects), by comparing hemodialysis and nonhemodialysis days. They compared

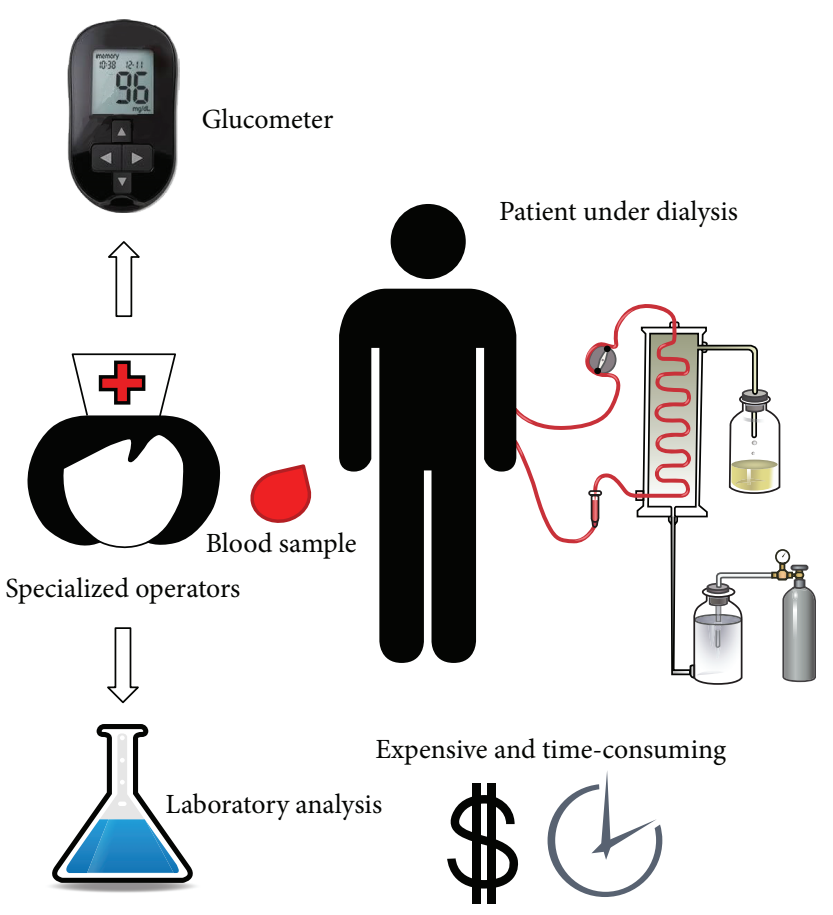

FIGURE 1: Schematic representation of the traditional approach for glucose measurement during dialysis.

some strip sensors, representing different technology formats: the StatStrip (Nova Biomedical, Waltham, MA, USA) glucose strip, a modified glucose oxidase-based amperometric test system with hematocrit and chemical interference corrections; Accu-Chek Aviva (Roche Diagnostics, Indianapolis, IN, USA), a glucose dehydrogenase/coenzyme (pyrroloquinoline quinone) based amperometric strip; Freestyle (Abbott Diabetes Care, Alameda, CA, USA) with an electrochemical glucose dehydrogenase/coenzyme (nicotinamide adenine dinucleotide) based colorimetric strip; Bayer Elite XL (Bayer, Leverkusen, Germany), which again uses a glucose oxidase-based amperometric detection system. They compared the results to a standard medical laboratory analyzer for measuring glucose, assumed as the reference: the Dimension RxL analyzer (Dade Behring, Deerfield, IL, USA), which uses a plasma hexokinase method. They tested the samples at various daily times before and during dialysis: 06.00 (wakeup time), 09.00 (before dialysis), 11.00 (2 hours after starting dialysis session), and 13.00 (at the end of the session). During dialysis, blood samples were obtained from the blood tubing at both arterial and venous sites of the dialyzer. Similar procedures were also adopted in the study [15]. In [16], glucose concentrations were measured in fresh peritoneal dialysis solutions before and after the addition of glucose, to investigate the effect of $\mathrm{pH}$ and glucose concentration on sodium removal; glucose concentrations were analyzed using a Hitachi 717 instrument (Hitachi, Tokyo, Japan).

Icodextrin scored one of the most significant steps forward in peritoneal dialysis. It is a solution where glucose is replaced by a mixture of glucose polymers (maltodextrins) 
with various molecular weights, which generates ultrafiltration with a colloid osmotic mechanism comparable to that of glucose. The dialysis solution has usually an icodextrin concentration of 7.5\%, pH 5.0, and low content of glucose degradation products. The production of ultrafiltration is slower compared to that of glucose but prolonged in time. This solution has considerable advantages in long stasis, allowing a higher level of ultrafiltration even when there is peritonitis, or ultrafiltration deficit. However, icodextrin has also some disadvantages: as an example, it can induce increased transaminases, alkaline phosphatase, and decreased serum sodium, and it can interfere with the assay of blood glucose [17]. For this reason, some diabetic subjects under peritoneal dialysis using icodextrin have been reported to develop clinical hypoglycemia caused by incorrectly measured high level of blood glucose, with consequent excessive administration of insulin. This was because of the fact that most of the glucose test strips use glucose dehydrogenase pyrroloquinoline quinone (GDH-PQQ) for the reaction. Therefore, the maltose and maltotriose, which is degraded from icodextrin by amylase, are falsely detected as glucose, resulting in an erroneously high glucose level $[18,19]$.

To avoid this drawback different approaches have been proposed by some studies. In [19], glycemia measurement was compared in peritoneal dialysis subjects, using the standard laboratory reference method and two glucose selfmonitoring sensor systems, based on glucose dehydrogenase nicotinamide adenine dinucleotide (GDH-NAD, Optium Xceed, Abbott Laboratories, Abbott Park, IL, USA) and traditional GDH-PQQ (Accu-Chek Active, Roche Diagnostics, Indianapolis, IN, USA), respectively. Results showed that the serum glucose levels, in subjects using icodextrin solution, were more accurately measured by the GDH-PQQ-based glucose self-monitoring sensor system. Thus, GDH-PQQbased systems should be preferred to avoid misinterpretation of hyperglycemia and subsequent overinjection of insulin. In [20], Perera et al. analyzed in vitro and in vivo blood samples, in subjects under icodextrin-based dialysis, using the hexokinase NADP (nicotinamide adenine dinucleotide phosphate) laboratory reference method (Modular-PPE, Roche Diagnostics, Indianapolis, IN, USA) compared with five glucose sensor systems: Accu-Chek Advantage-II and Accu-Chek Performa (Roche Diagnostics, Mannheim, Germany) based on GDH-PQQ strips, two Optium Xceed devices (Abbott Diabetes Care, Alameda, CA, USA) with glucose dehydrogenase-NAD $5 \mathrm{~s}$ and $20 \mathrm{~s}$ strips, and StatStrip (Nova Biomedical, Waltham, MA, USA), again with glucose oxidase-NAD strips. Four meters provided plasma equivalent glucose readings, whereas Accu-Chek AdvantageII provided whole-blood glucose readings. In general all the icodextrin studies concluded that glucose sensors using the more glucose-specific glucose dehydrogenase-NAD enzyme systems were less affected by icodextrin effects compared to sensors using the GDH-PQQ-based system, with differences between the two methods that were clinically significant, with a bias of $>10 \%$ for each examined metabolite of icodextrin (the effect being additive) [19, 20].

Few other studies dealt with the measurement of glucose in the dialysate. The most significant was by Deman et al. [21], where a small planar amperometric device produced with standard thick film technology was employed as sensor. In that device, the work and counter electrode were screenprinted with $\mathrm{RuO} 2$ paste. A mixture of the glucose oxidase enzyme (GOx) from Aspergillus niger, $150 \mathrm{U} \mathrm{mg}^{-1}$, bovine serum albumin (BSA) Fraction V, and glutardialdehyde was dispensed on the silanized electrode surface; after $24 \mathrm{~h}$ the enzyme membrane was cross-linked and dip-coated with a polyvinyl butyral diffusion membrane. The enzyme stability of the sensor was high; thus the sensor could be stored for several weeks without special requirements. The sensor could be plugged directly into the output line of a standard dialyzer, and the signal was transmitted to a low power multifunctioning personal computer. The sensor could be used for 3-4 hours in the normal dialysate set-up. The system was userfriendly, and hence the clinical personnel found no relevant problems in monitoring the measurements process.

\section{Glucose Determination with Continuous Glucose Monitoring (CGM) Technology}

Apart from possible accuracy problems, the main limitations in the use of a traditional approach for glucose determination are that (a) consumables are typically required, possibly for each measure: if the measurement is performed several times during the dialysis session, this may result in a relevant expense; (b) in any case, the measurement is discontinuous; thus some possible rapid glucose changes (e.g., leading to a hypoglycemic condition) may not be revealed promptly; (c) for every measure, the action of an operator (nurse or similar figure) is required to get the sample, and this again may have effects on the cost of the dialysis session. It should be noted that sometimes the sample (plasma ultrafiltrate solution, or dialysate) is collected for measurements of several compounds, including glucose, through a hemogas analyzer [22]; thus the need of the operator action could not be attributed to glucose determination exclusively. At any rate, limitation of discontinuous determination remains. This is one of the reasons why in many studies glucose measurement during dialysis has been performed through continuous glucose monitoring (CGM) technology (Figure 2). In fact, the glucose information delivered by CGM potentially allows for more useful therapeutic decisions aimed at optimizing glucose levels.

The CGM devices available today can be classified into those which measure real-time glucose levels (RT-CGM) and those which record glucose levels on a continuous basis but only allow retrospectively access to the information (r-CGM), after data are downloaded from the device.

The first system for continuous glucose monitoring approved by the US Food and Drug Administration (FDA) was the MiniMed CGMS, The Continuous Glucose Monitoring System (CGMS) developed by Medtronic (Dublin, Ireland) $[23,24]$. The sensor is a glucose oxidase-based platinum electrode that is inserted through an attachment needle into the subcutaneous tissue of the anterior abdominal wall, or other appropriate body sites of the patient, using a springloaded device (the Sen-serter). Glucose oxidase catalyzes the oxidation of glucose in the interstitial fluid, which generates 


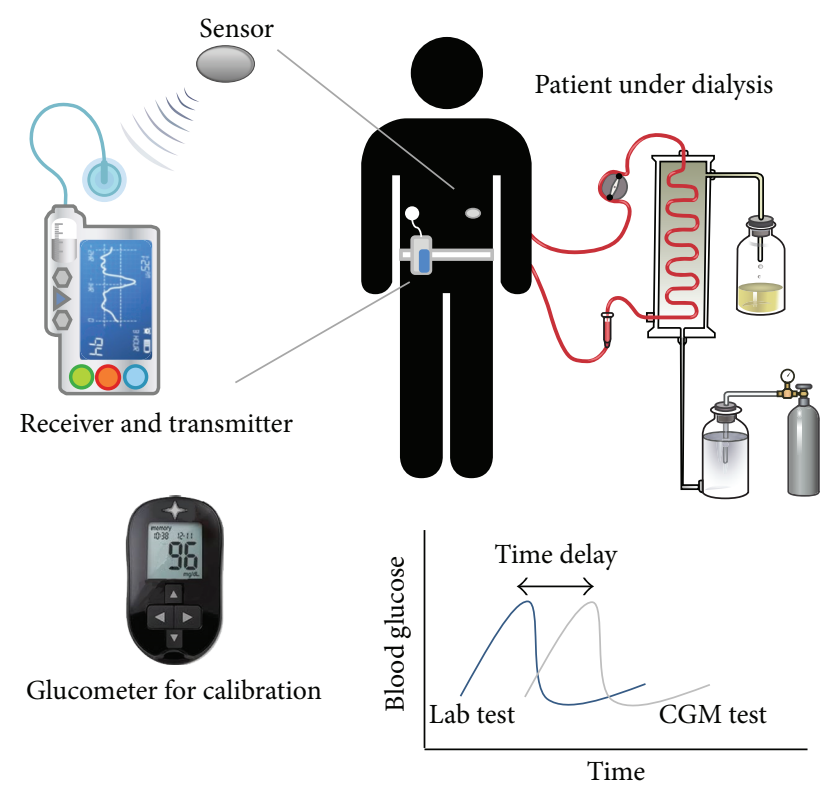

FIgURE 2: Schematic representation of the CGM approach for glucose measurement during dialysis.

an electrical current. The current is carried by a cable to a small monitor $(14.4 \times 18.0 \times 9.4 \mathrm{~cm})$ that analyzes the data every $10 \mathrm{~s}$ and reports average values every $5 \mathrm{~min}$, giving a total of 288 readings per day. Sensor readings are calibrated against capillary blood glucose measurements obtained with conventional self-monitoring blood glucose meters [25]. The sensor is a r-CGM type and does not provide real-time glucose values to the wearer; data can only be downloaded by clinicians after measurements. The studies that we analyzed [25-30] all used almost minor variations of this type of sensor, except for the studies $[31,32]$, which used instead GlucoDay CGM device from A. Menarini Diagnostics (Florence, Italy).

In all studies [25-30], the interstitial glucose range was $40-400 \mathrm{mg} / \mathrm{dL}$, and each sensor was used continuously for up to 72 hours. However, the calibration process was slightly different among the different studies. To calibrate the CGMS readings, in studies $[25,26,28,30]$ subjects were asked to perform finger-stick blood-sugar measurements using a traditional blood glucose meter at least 4 times daily; also, in study [28] it was reported to take two of the measurements before breakfast and before dinner. In [27], for calibration purpose three capillary blood glucose readings were entered in the CGMS memory at prescribed intervals. In [29], the CGMS was calibrated only to a limited glucose level, and then subjects were instructed to perform additional capillary glucose measurements.

In the studies $[31,32]$, the GlucoDay was used instead. It is a portable instrument provided with a micropump and a biosensor coupled to a microdialysis system capable of recording the subcutaneous glucose level every $3 \mathrm{~min}$. The system has the following main features: (a) a programmable micropump with $15-100 \mu \mathrm{ml} / \mathrm{min}$ flow rate; (b) a fluidic line made of nylon in all the sections except in the peristaltic pump; (c) a wall jet flow cell made of a platinum electrode and a three-layer membrane system; (d) a microcontroller for pump speed programming, signal acquisition, and data storage (12-bit resolution, 1800 data points); (e) 32-alphanumericcharacter display and a keyboard; (f) RS232 interface at 1200 baud for downloading the data to a personal computer; (g) a $9 \mathrm{~V}$ battery for $48 \mathrm{~h}$ continuous data recording. Two different flow rates can be used: a faster one for filling and/or washing the line and a slower one for the continuous feeding of the microdialysis probe. Two plastic bags, one for the buffer solution and one for the wasted solution, complete the apparatus, which for its dimension and weight can be contained in a pouch and worn as a belt.

Early generation r-CGM devices were relatively large and required a major degree of interaction by the subject, while the latest CGM devices are smaller, light, and almost invisible under clothes, thus causing less stress to the subjects. Ipro 2 is the successor of the MiniMed 530G, first-generation artificial pancreas device system approved by FDA. The device can automatically stop insulin delivery when glucose values reach a preset level and when the patient does not respond to the Threshold Suspend alarm. Finger-prick blood glucose readings are still required for calibration but these do not need to be entered by the patient into the device: the calibration readings are integrated with the raw CGM data at the time of the download via a personal computer, which is usually performed by a health professional. Ipro 2 was used in studies [33-35]. Subjects were trained to measure capillary blood glucose 3-4 times/day (depending on the study) and to record glucose values and administered insulin dosages, as well as details of food consumption, physical activity, and symptoms. In the mentioned studies, CGM data were also used to represent the glucose profile of a typical day with or without dialysis.

The other two CGM devices of the last generation were used in a few studies: Navigator (Abbott, Rungis, France) in studies [36, 37] and DexCom SEVEN PLUS (DexCom, CA, USA) in study [38]. Technologies, operations, and calibration procedures of these devices are similar to those described above.

All the mentioned studies concluded that in subjects under dialysis treatment CGM improves glycemic control and decreases the hypoglycemic events. Also, in these types of subjects CGM was proved useful for the management of insulin therapy. However, there are still some limitations, and positive results need to be confirmed by long-term studies with larger number of subjects.

\section{Glucose Determination with Optical Sensors}

The CGM approach overcomes some limitations of the traditional glucose measurement. However, the main disadvantage of CGM is that glucose determination is performed in the interstitial fluid, and not in plasma. It is known that though glucose levels in plasma and interstitial fluid are obviously related, there may be a temporal delay between glucose levels in the latter compared to the former [39-41]. Thus, in a specific, critical condition like the dialysis treatment, this may be a severe limitation, especially in the case of rapid changes in glucose values. Thus, ideally, glucose determination should 


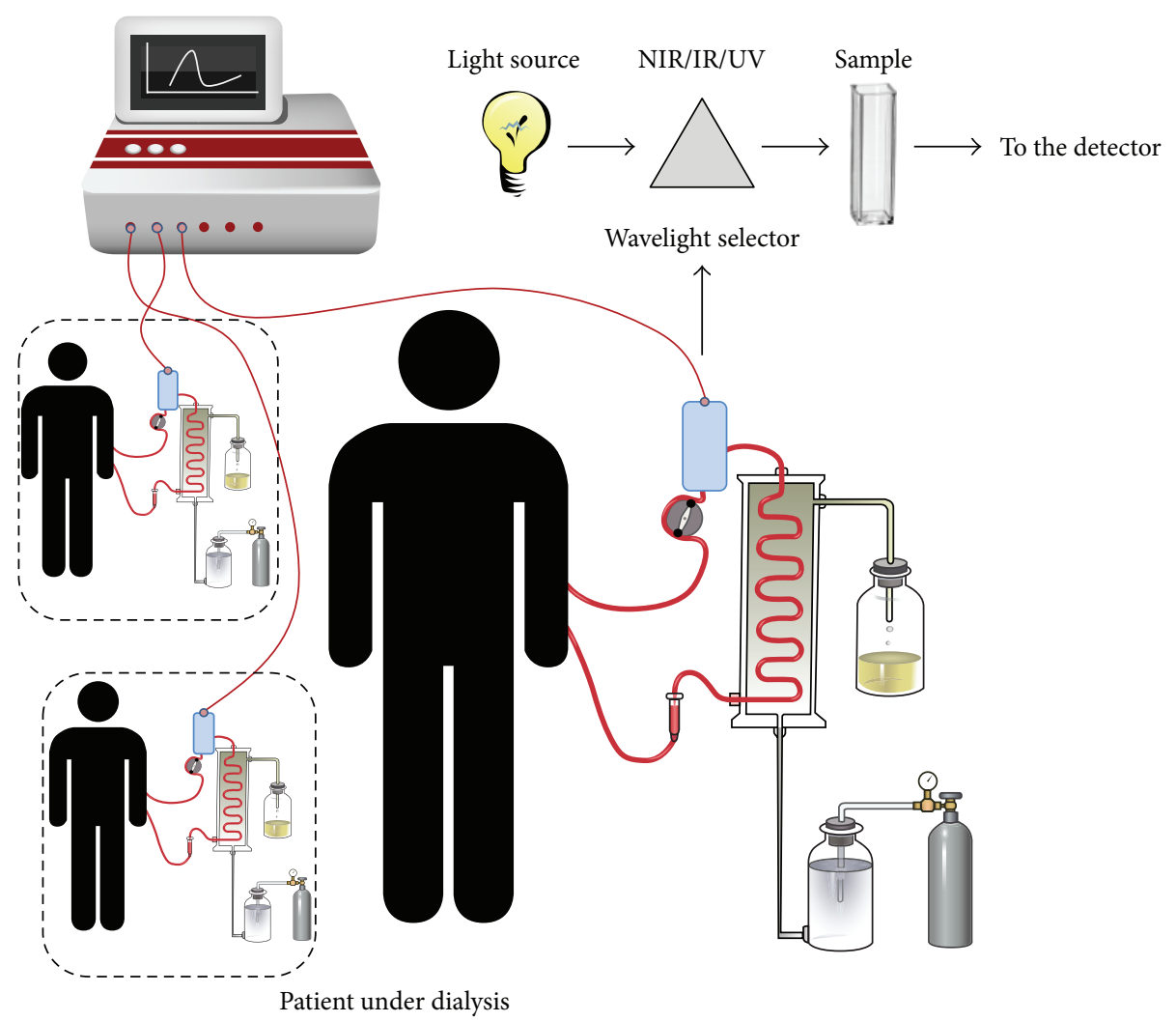

FIGURE 3: Schematic representation of the optical approach for glucose measurement during dialysis. Ideally, glucose measures in different subjects may be possible with a single spectrophotometer.

be continuous, similarly to the CGM approach, but possibly in plasma. During a hemodialysis session, plasma is already circulating outside the patient's body, and hence glucose determination with an optical method may be simpler than similar determination in intact body, during ordinary life. In fact, some strategies for noninvasive glucose determination, based on optical approaches, have been proposed, but, to our knowledge, a satisfactory solution has not been found yet [42, 43], probably due to several possible confounding factors. In the case of glucose determination during hemodialysis, due to the extracorporeal blood circulation several confounding factors may not be present, and thus an optical approach may be successful. Indeed, though a few, some studies presented an optical approach to this purpose (Figure 3).

In the study [44], Roth et al. proposed a method for monitoring a hemodialysis session based on quantitative infrared spectroscopic (IR) determination of molecules from patient's blood. For the quantitative determination of the constituents of interest over the period of hemodialysis sessions (up to $5 \mathrm{~h}$ ), authors of study [44] adapted a compact instrument with adequate sensitivity and stability (model Alpha, Bruker Optics, Ettlingen, Germany). Data acquisition, Fourier transformation, and spectral processing were performed using appropriate software (Bruker software program OPUS version 6.5). The calibration procedure was based on artificial dialysate samples, prepared from stock solutions with known concentrations, and real samples taken from the subjects during the hemodialysis sessions. To optimize calibration, all the samples were previously analyzed by conventional clinical chemistry. The IR spectra of urea, glucose, lactate, and creatinine (as the major components in dialysate) exhibited several bands in the $2000-800 \mathrm{~cm}^{-1}$ range, although they were partly overlapping and thus necessitated the use of multivariate analysis methods. The proposed monitoring approach of the hemodialysis session, including glucose determination, can be performed in real time and in line, directly at the dialysis machine; it requires little human intervention and no consumables at all. Authors of study [44] claimed to have started a clinical trial, but in [44] they reported results on two patients only.

In studies $[45,46]$ near-infrared spectroscopy (NIR) was used for measuring different metabolites during dialysis. In [45], NIR was proposed as a method for providing realtime urea concentrations during hemodialysis treatment. In this investigation, the three major regions of the NIR spectrum were evaluated for the selective measurement of urea in the basic matrix of the dialysate solution. The best analytical performance was found for the measurements within the spectral range that extended from 5000 to $4000 \mathrm{~cm}^{-1}(2.0-2.5 \mathrm{~mm})$, collected with a Nicolet Magna 550 Fourier transform infrared spectrometer (Nicolet Analytical Instruments, WI, USA). This spectrometer was equipped with a standard 20-watt tungsten source, a calcium-fluoride beam splitter, and a cryogenically cooled indium antimonide 
TABLE 1: Basic information on the analyzed studies on glucose determination during dialysis treatment; PD: peritoneal dialysis; HD: hemodialysis; D: dialysis; pre-D: predialysis. Of note, some studies include subjects not under dialysis treatment.

\begin{tabular}{lccl}
\hline Approach & $\begin{array}{c}\text { Number of } \\
\text { articles }\end{array}$ & $\begin{array}{c}\text { Total number } \\
\text { of subjects }\end{array}$ & Type of subjects \\
\hline Traditional & 8 & 392 & ${ }^{*} 155$ in PD, ${ }^{*} 180$ in HD, and ${ }^{*} 57$ in pre-D \\
\hline CGM & 14 & 431 & $\begin{array}{l}230 \text { diabetic subjects in HD, 13 nondiabetic subjects (not in dialysis), } \\
36 \text { diabetic subjects in pre-D, } 68 \text { type } 1 \text { diabetic subjects (not in } \\
\text { dialysis), 19 nondiabetic subjects in PD, 39 type 2 diabetic subjects } \\
\text { (not in dialysis), and 26 diabetic subjects in PD }\end{array}$ \\
\hline Optical & 4 & 39 & 5 diabetic subjects in HD, ${ }^{*} 37$ subjects in HD \\
\hline
\end{tabular}

* In these subjects undergoing dialysis the possible presence of diabetic condition is not specified.

detector. Detection was restricted to the indicated spectral region by positioning an interference filter $(2.0-2.5 \mathrm{~mm}$; Barr Associates, MA, USA) immediately before the sample. Selectivity for urea over glucose was examined as a step in the evaluation of this scheme for measuring urea accurately in the dialysate samples, collected during the dialysis treatment. Glucose determination was performed because it is known that glucose concentration in dialysate samples can vary in accordance to the difference between its concentration in the patient's blood and in the original dialysate fluid (glucose is added to the original dialysate solution to avoid hypoglycemia). The results showed that selective urea measurements were possible in solutions with glucose variations of 1-35 mmol/L. From our point of view, the study [45] showed that glucose determination by NIR is possible, though no detailed results were reported, the focus being on urea.

In the study [46], NIR was employed for the simultaneous determination of the concentrations of glucose and lactic acid in peritoneal dialysis solutions. The sample solution, which was a peritoneal dialysis solution, was analyzed with a near-infrared spectrophotometer (NIRS6500SPL, Nireco, Ishikawa-cho, Hachioji, Tokyo, Japan). The absorbance from 400 to $2500 \mathrm{~nm}$ was measured at $2 \mathrm{~nm}$ intervals. The cell holder was maintained at a constant temperature by circulating water from the water bath throughout the jacket of the cell holder. The spectrum of each sample was obtained by taking the average reading of 32 scans. However, the results, while showing a good correlation with the enzymatic reference method, focused on glucose levels outside the physiological range (10-70 g/L), and at rather distant experimental points.

The absorption of ultraviolet (UV) radiation was used by Fridolin et al. in [47] to monitor solutes in a spent dialysate. For the determination of UV-absorbance, a double-beam spectrophotometer (UVIKON 943, Kontron, Italy) with an accuracy of $\pm 1 \%$ was used. The most essential parts of the optics module incorporated into the spectrophotometer are (a) a light source, (b) a monochromator to resolve the source of radiation into component "monochromatic" elements, and (c) a light sensor to detect the radiation after passing the optical cuvette containing the solution under study. Measurements were performed both on collected dialysate samples and during on-line monitoring; the spectrophotometer was connected to the fluid outlet of the dialysis machine, with all spent dialysate passing through a specially designed cuvette for optical single-wavelength measurements. The concentrations of the substances of interest were also determined using reference laboratory techniques and, based on the UV-absorbance results, correlation coefficients were calculated. The experimental results indicated a good correlation between UV-absorbance and the laboratory reference for several small solutes such as urea, creatinine, and uric acid, at a fixed wavelength of $285 \mathrm{~nm}$, though it should be noted that measurements of different compounds at fixed wavelength may suffer for selectivity problems. Worse results were achieved for glucose and other metabolites (sodium, calcium, vitamin $B_{12}$, and albumin). Therefore, UV approach, though theoretically possible, with the current technology appears to be not sufficiently accurate for glucose determination during dialysis session.

\section{Conclusions}

In this review study, we have analyzed the sensors, technologies, and approaches for glucose determination during dialysis treatment. Table 1 summarizes some information for the studies analyzed.

We can conclude that (a) as regards the traditional approach, if we refer to the classical laboratory analysis methods, they are definitely the gold standard in terms of quality, precision, and reliability of the information provided, but on the other hand the measurement procedure is slow and cumbersome, it does not provide continuous and realtime data, and it can be expensive due to the need of complex equipment and qualified personnel at different levels (nurses, laboratory technicians); if we refer to the use of sensors and devices for self-monitoring of blood glucose, they are not optimal in terms of the quality of the measurement, and, in the specific context of a dialysis session, they still require the intervention of a nurse or other medical professionals; also, they require consumables; (b) the CGM approach represents a step forward compared to traditional approaches: it allows continuous, possibly real-time glucose measurement, and it may require somehow lower intervention of the medical personnel; however, the measure does not take place in plasma but in the interstitial fluid, and some delay in the glucose level is typically present; (c) the optical approach is still largely unexplored and from a technological point of view it has not yet been developed to its full potential. On the other hand, it 
appears a promising approach, since it can lead to continuous measurement of glucose in plasma (and not in the interstitial fluid), with small or no requirement of consumables, and with the advantage of modest needs in terms of intervention of the medical personnel.

In conclusion, optical methods may become the most convenient approach for glucose measurement during dialysis treatment, but in the light of the small number of studies reported in the literature, further studies are needed to deeply understand the potential, and possible limitations, of these approaches.

\section{Conflict of Interests}

The authors declare that there is no conflict of interests regarding the publication of this paper.

\section{References}

[1] G. Trifirò, J. Sultana, F. Giorgianni et al., "Chronic kidney disease requiring healthcare services: a new approach to evaluate epidemiology of renal disease," BioMed Research International, vol. 2014, Article ID 268362, 6 pages, 2014.

[2] F. X. Liu, P. Rutherford, K. Smoyer-Tomic, S. Prichard, and S. Laplante, "A global overview of renal registries: a systematic review," BMC Nephrology, vol. 16, article 31, 2015.

[3] T. Oomichi, M. Emoto, T. Tabata et al., "Impact of glycemic control on survival of diabetic patients on chronic regular hemodialysis: a 7-year observational study," Diabetes Care, vol. 29, no. 7, pp. 1496-1500, 2006.

[4] T. Morioka, M. Emoto, T. Tabata et al., "Glycemic control is a predictor of survival for diabetic patients on hemodialysis," Diabetes Care, vol. 24, no. 5, pp. 909-913, 2001.

[5] G. Spasovski, R. Vanholder, B. Allolio et al., "Clinical practice guideline on diagnosis and treatment of hyponatraemia. Hyponatraemia Guideline Development Group," Nephrology Dialysis Transplantation, vol. 29, supplement 2, pp. il-i39, 2014.

[6] L. Monnier and C. Colette, "Glycemic variability: should we and can we prevent it?" Diabetes Care, vol. 31, supplement 2, pp. S150-S154, 2008

[7] M. Abe and K. Kalantar-Zadeh, "Haemodialysis-induced hypoglycaemia and glycaemic disarrays," Nature Reviews Nephrology, vol. 11, no. 5, pp. 302-313, 2015.

[8] S. Korsatko, M. Ellmerer, L. Schaupp et al., "Hypoglycaemic coma due to falsely high point-of-care glucose measurements in an ICU-patient with peritoneal dialysis: a critical incidence report," Intensive Care Medicine, vol. 35, no. 3, pp. 571-572, 2009.

[9] E. Disse and C. Thivole, "Hypoglycemic coma in a diabetic patient on peritoneal dialysis due to interference of icodextrin metabolites with capillary blood glucose measurements," Diabetes Care, vol. 27, no. 9, p. 2279, 2004.

[10] T. W. Mak, C. K. Cheung, C. M. Cheung, C. B. Leung, C. W. Lam, and K. N. Lai, "Interference of creatinine measurement in CAPD fluid is dependent on glucose and creatinine concentrations," Nephrology Dialysis Transplantation, vol. 12, pp. 184-186, 1997.

[11] M. Kishimoto and M. Noda, "Glucose management in diabetic patients undergoing hemodialysis," Diabetology International, vol. 5, no. 2, pp. 84-91, 2014.
[12] C. J. Wason, J. Green, C. Miller, and D. M. Roxe, "Reagent strip glucose monitoring methods in chronic hemodialysis," Diabetes Care, vol. 8, no. 6, pp. 603-604, 1985.

[13] B. H. Ginsberg, "Factors affecting blood glucose monitoring: sources of errors in measurement," Journal of Diabetes Science and Technology, vol. 3, no. 4, pp. 903-913, 2009.

[14] B. Bewley, S. O'Rahilly, R. Tassell, J. DuBois, and E. Donald, "Evaluation of the analytical specificity and clinical application of a new generation hospital-based glucose meter in a dialysis setting," Point of Care, vol. 8, no. 2, pp. 216-221, 2009.

[15] M. Abe, K. Kaizu, and K. Matsumoto, "Evaluation of the hemodialysis-induced changes in plasma glucose and insulin concentrations in diabetic patients: comparison between the hemodialysis and non-hemodialysis days," Therapeutic Apheresis and Dialysis, vol. 11, no. 4, pp. 288-295, 2007.

[16] V. La Milia, S. Di Filippo, M. Crepaldi et al., "Spurious estimations of sodium removal during CAPD when $[\mathrm{Na}]^{+}$is measured by Na electrode methodology," Kidney International, vol. 58, no. 5, pp. 2194-2199, 2000.

[17] C. Fourtounas, A. Hardalias, P. Dousdampanis, B. Papachristopoulos, E. Savidaki, and J. G. Vlachojannis, "Sodium removal in peritoneal dialysis: the role of icodextrin and peritoneal dialysis modalities," Advances in Peritoneal Dialysis, vol. 24, pp. 27-31, 2008.

[18] C. A. Firanek, D. T. Jacob, and J. A. Sloand, "Avoidable iatrogenic hypoglycemia in patients on peritoneal dialysis: the risks of nonspecific glucose monitoring devices and drug-device interaction," Journal of Patient Safety, vol. 10, no. 4, pp. 218-221, 2014.

[19] C.-Y. Tsai, S.-C. Lee, C.-C. Hung et al., "False elevation of blood glucose levels measured by GDH-PQQ-based glucometers occurs during all daily dwells in peritoneal dialysis patients using Icodextrin," Peritoneal Dialysis International, vol. 30, no. 3, pp. 329-335, 2010.

[20] N. J. Perera, P. M. Stewart, P. F. Williams, E. L. Chua, D. K. Yue, and S. M. Twigg, "The danger of using inappropriate point-ofcare glucose meters in patients on icodextrin dialysis," Diabetic Medicine, vol. 28, no. 10, pp. 1272-1276, 2011.

[21] P. Deman, J. Suls, and W. Sansen, "Continuous differential monitoring of the spent dialysate glucose level: clinical evaluation," Sensors and Actuators B: Chemical, vol. 44, no. 1-3, pp. 304-308, 1997.

[22] A. Tura, S. Sbrignadello, E. Mambelli, P. Ravazzani, A. Santoro, and G. Pacini, "Sodium concentration measurement during hemodialysis through ion-exchange resin and conductivity measure approach: in vitro experiments," PLoS ONE, vol. 8, no. 7, Article ID e69227, 2013.

[23] S. Vaddiraju, D. J. Burgess, I. Tomazos, F. C. Jain, and F. Papadimitrakopoulos, "Technologies for continuous glucose monitoring: current problems and future promises," Journal of Diabetes Science and Technology, vol. 4, no. 6, pp. 1540-1562, 2010.

[24] D. J. O’Neal and A. Jenkins, "Continuous glucose monitoring: comparing. Real-time and retrospective devices," Infusystems USA, vol. 7, pp. 26-30, 2010.

[25] H. S. Jung, H. I. I. Kim, M. J. Kim et al., "Analysis of hemodialysis-associated hypoglycemia in patients with type 2 diabetes using a continuous glucose monitoring system," Diabetes Technology and Therapeutics, vol. 12, no. 10, pp. 801-807, 2010.

[26] A. Skubala, J. Zywiec, K. Zełobowska, J. Gumprecht, and W. Grzeszczak, "Continuous glucose monitoring system in 72hour glucose profile assessment in patients with end-stage renal 
disease on maintenance continuous ambulatory peritoneal dialysis," Medical Science Monitor, vol. 16, no. 2, pp. CR75-CR83, 2010.

[27] E. Sobngwi, G. Ashuntantang, E. Ndounia et al., "Continuous interstitial glucose monitoring in non-diabetic subjects with end-stage renal disease undergoing maintenance haemodialysis," Diabetes Research and Clinical Practice, vol. 90, no. 1, pp. 22-25, 2010.

[28] J.-P. Riveline, J. Teynie, S. Belmouaz et al., "Glycaemic control in type 2 diabetic patients on chronic haemodialysis: use of a continuous glucose monitoring system," Nephrology Dialysis Transplantation, vol. 24, no. 9, pp. 2866-2871, 2009.

[29] J. Marshall, P. Jennings, A. Scott, R. J. Fluck, and C. W. Mcintyre, "Glycemic control in diabetic CAPD patients assessed by continuous glucose monitoring system (CGMS)," Kidney International, vol. 64, no. 4, pp. 1480-1486, 2003.

[30] W. D. Schwing, P. Erhard, L. N. Newman et al., "Assessing 24-hour blood glucose patterns in diabetic paitents treated by peritoneal dialysis," Advances in Peritoneal Dialysis, vol. 20, pp. 213-216, 2004.

[31] S. Kazempour-Ardebili, V. L. Lecamwasam, T. Dassanyake et al., "Assessing glycemic control in maintenance hemodialysis patients with type 2 diabetes," Diabetes Care, vol. 32, no. 7, pp. 1137-1142, 2009.

[32] M. Mirani, C. Berra, S. Finazzi et al., "Inter-day glycemic variability assessed by continuous glucose monitoring in insulintreated type 2 diabetes patients on hemodialysis," Diabetes Technology and Therapeutics, vol. 12, no. 10, pp. 749-753, 2010.

[33] T. Mori, M. Chida, I. Oba et al., "Diurnal variations of blood glucose by continuous blood glucose monitoring in peritoneal dialysis patients with diabetes," Advances in Peritoneal Dialysis, vol. 30, pp. 54-59, 2014.

[34] M. Joubert, C. Fourmy, P. Henri, M. Ficheux, T. Lobbedez, and Y. Reznik, "Effectiveness of continuous glucose monitoring in dialysis patients with diabetes: the DIALYDIAB pilot study," Diabetes Research and Clinical Practice, vol. 107, pp. 348-354, 2015.

[35] M. Gai, I. Merlo, S. Dellepiane et al., "Glycemic pattern in diabetic patients on hemodialysis: continuous glucose monitoring (CGM) analysis," Blood Purification, vol. 38, no. 1, pp. 68-73, 2014.

[36] L. Képénékian, A. Smagala, L. Meyer et al., "Continuous glucose monitoring in hemodialyzed patients with type 2 diabetes: a multicenter pilot study," Clinical Nephrology, vol. 82, no. 4, pp. 240-246, 2014.

[37] F. Chantrel, H. Sissoko, L. Képénékian et al., "Influence of dialysis on the glucose profile in patients with diabetes: usefulness of continuous glucose monitoring," Hormone and Metabolic Research, vol. 46, no. 11, pp. 810-813, 2014.

[38] S. Popa, C. Văduva, M. Moţa, and E. Moţa, "Peritoneal dialysis-risk factor for glycemic variability assessed by continuous glucose monitoring system," Romanian Journal of Diabetes Nutrition and Metabolic Diseases, vol. 21, no. 1, pp. 47-54, 2014.

[39] P. Rossetti, J. Bondia, J. Vehí, and C. G. Fanelli, "Estimating plasma glucose from interstitial glucose: the issue of calibration algorithms in commercial continuous glucose monitoring devices," Sensors, vol. 10, no. 12, pp. 10936-10952, 2010.

[40] E. Kulcu, J. A. Tamada, G. Reach, R. O. Potts, and M. J. Lesho, "Physiological differences between interstitial glucose and blood glucose measured in human subjects," Diabetes Care, vol. 26, no. 8, pp. 2405-2409, 2003.
[41] K. Rebrin, N. F. Sheppard Jr., and G. M. Steil, "Use of subcutaneous interstitial fluid glucose to estimate blood glucose: revisiting delay and sensor offset," Journal of Diabetes Science and Technology, vol. 4, no. 5, pp. 1087-1098, 2010.

[42] A. Tura, A. Maran, and G. Pacini, "Non-invasive glucose monitoring: assessment of technologies and devices according to quantitative criteria," Diabetes Research and Clinical Practice, vol. 77, no. 1, pp. 16-40, 2007.

[43] S. K. Vashist, "Non-invasive glucose monitoring technology in diabetes management: a review," Analytica Chimica Acta, vol. 750, pp. 16-27, 2012.

[44] A. Roth, F. Dornuf, O. Klein, D. Schneditz, H. Hafner-Gießauf, and W. Mäntele, "Infrared spectroscopy in hemodialysis: reagent-free monitoring of patient detoxification by infrared spectroscopy," Analytical and Bioanalytical Chemistry, vol. 403, no. 2, pp. 391-399, 2012.

[45] C. V. Eddy and M. A. Arnold, "Near-infrared spectroscopy for measuring urea in hemodialysis fluids," Clinical Chemistry, vol. 47, pp. 1279-1286, 2001.

[46] T. Yano, H. Matsushige, K. Suehara, and Y. Nakano, "Measurement of the concentrations of glucose and lactic acid in peritoneal dialysis solutions using near-infrared spectroscopy," Journal of Bioscience and Bioengineering, vol. 90, no. 5, pp. 540$544,2000$.

[47] I. Fridolin, M. Magnusson, and L.-G. Lindberg, "On-line monitoring of solutes in dialysate using absorption of ultraviolet radiation: technique description," International Journal of Artificial Organs, vol. 25, no. 8, pp. 748-761, 2002. 


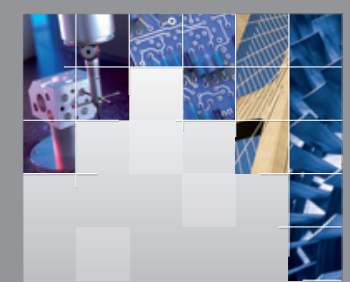

\section{Enfincering}
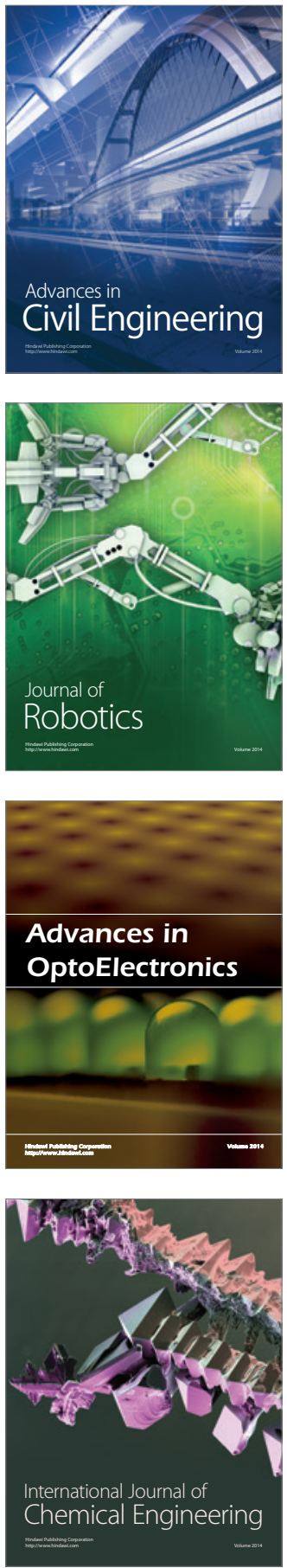

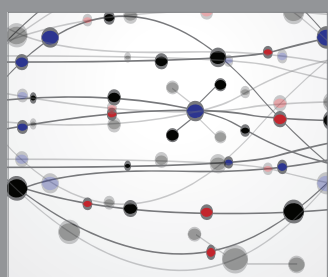

The Scientific World Journal

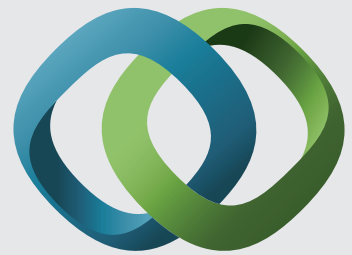

\section{Hindawi}

Submit your manuscripts at

http://www.hindawi.com
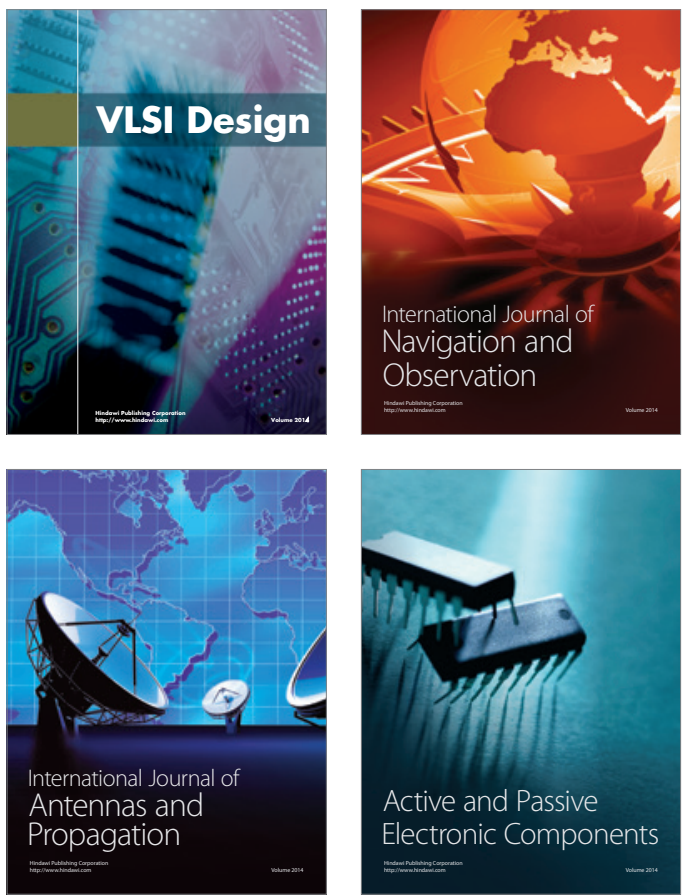
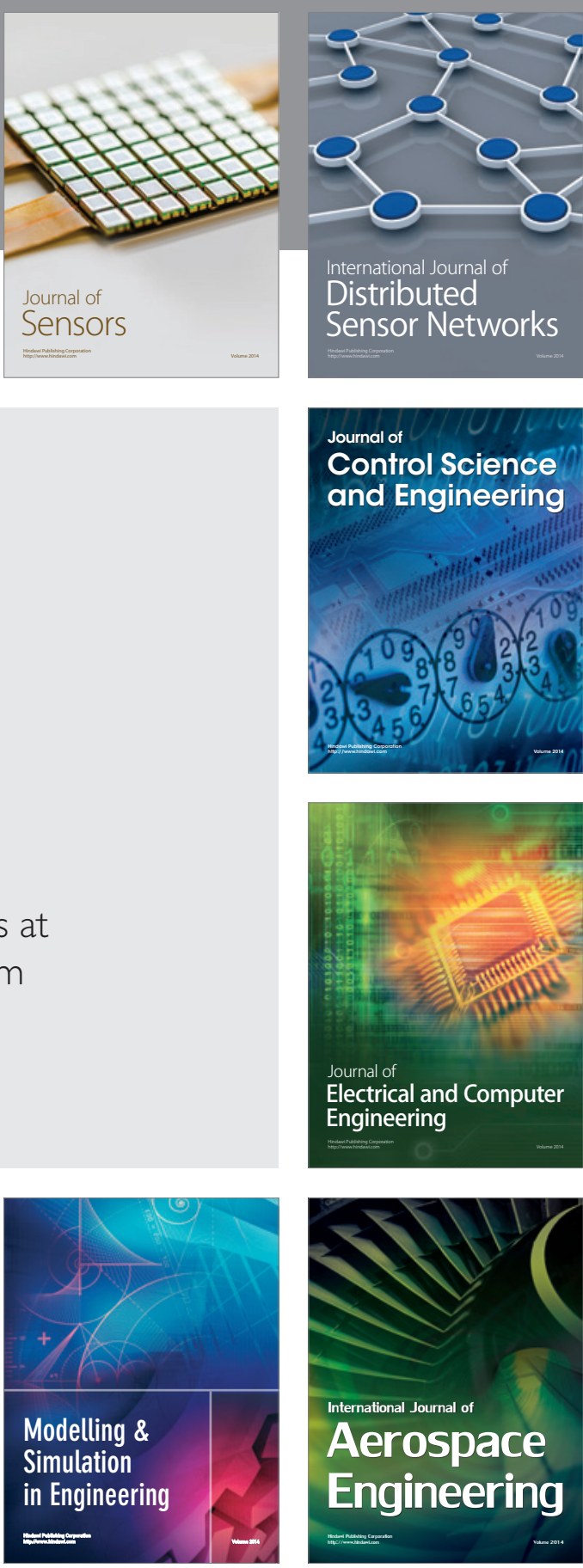

International Journal of

Distributed

Sensor Networks

Journal of

Control Science

and Engineering
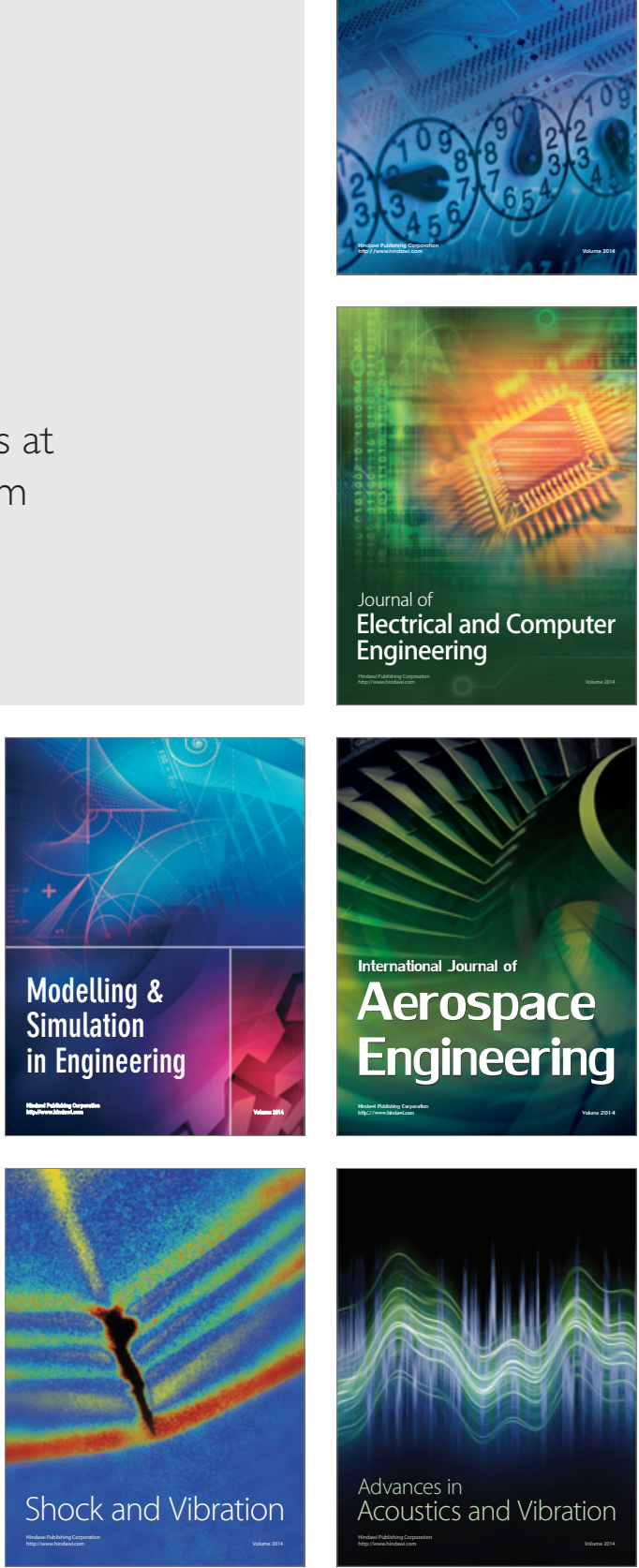